\title{
Digital Primary Sources - Raum für primär digitale Angelegenheiten
}

\author{
Von Definitionen der Digital Humanities ÜBER DAS Digitale ÖKOSystem als \\ PRIMÄRQUELLE BIS ZUR ERHALTUNG VON DIGITALEN QUELLEN
}

\section{Gernot Hausar}

Universität Wien, Institut für Geschichte

gernot.hausar@gmail.com / gernot.hausar@univie.ac.at

https://orcid.org/0000-0001-5784-988X

Keywords: Internet, Digital Sources, Digital Archaeology, Digital Ecosystem, Digital as

Primary Source

\begin{abstract}
In scientific interaction of digital humanities scholars with the digital ecosystem the focus is more on the use of digital tools and less on the digital ecosystem as a primary source. This is made visible both by the range of definitions and the focus of scientific endeavors. This paper argues that the consequences of this might be the loss of valuable digital only sources and offers some possible approaches and „hacks" to mitigate the damage and facilitate scientific cooperation.
\end{abstract}

\section{Primär digitale Angelegenheiten ${ }^{1}$}

Primär digitale Quellen verändern gleich einem ,trojanischen Pferd' alle Abläufe geisteswissenschaftlicher Forschung nachhaltig. Ein Anzeichen für die sich stellenden Herausforderungen bietet beispielsweise die Suche nach gemeinsamen Definitionen des ,Digitalen', die in Summe mehr als seine einzelnen Komponenten darstellt und sich ,verlebendigt'. ${ }^{2}$ Denn während sich die Digital Humanities die digitalen Werkzeuge, Metadaten und Kommunikationstools bereits weitgehend zu eigen gemacht haben, steht eine gemeinsame Arbeitsdefinition des digitalen Ökosystems und des Digitalen als Raum, die mit den sich rasch ändernden Rahmenbedingungen mitwachsen kann, noch aus.

1 Basiert teilweise auf: Gernot HAUSAR, Weitere Überlegungen zu digitalen Geschichts- und Geisteswissenschaften, in: Julia Menzel, Hg., Digitalität - Theorien und Praktiken des Digitalen in den Geisteswissenschaften 2016, online unter: https://web.archive.org/web/20190409173203/https://digigeist.hypotheses.org/308 (09.04.2019). 
Einen Überblick über die Vielfalt an Definitionen gibt beispielsweise das Projekt „What is Digital Humanities?“3, welches Definitionen von Teilnehmer*innen des Projektes „Day of DH“4 2009-2014 gesammelt und dargestellt hat.

Dies macht es nötig, auch hier auf die gewählten Begriffe kurz einzugehen:

- „Digitales Ökosystem“ umfasst sowohl die zugrundeliegende physische Infrastruktur (z.B. Kabel, Eingabe- und Speichergeräte, Computerchips) als auch die darüber ausgetauschten bzw. lokal gespeicherten und verarbeiteten Daten.

- „Digitaler Raum“ beschreibt die im digitalen Ökosystem existenten Räume, die im Sinne von Henri Lefebvres ${ }^{5}$ Ansatz durch die Nutzer*innen „produziert“ werden.

- „Digital Humanities“ wiederum umfasst die Untersuchung aller menschlichen Aktivitäten aus Sicht der „Humanities“ (e.g. Geistes- \& Kultur-, Kunst- und Sozialwissenschaften) durch Wiederherstellen, Erhaltung \& Archivierung sowie Untersuchung digitaler Vorgänge und Kultur mit technischen Hilfsmitteln.

Der Begriff des Digitalen selbst umfasst hier sowohl das Ökosystem als auch die durch die Nutzer* innen geschaffenen Räume. Diese Räume ermöglichen es uns, in rein digitalen Umgebungen Artefakte zu erschaffen, die in vollem Umfang nur in dieser Umgebung Bestand haben können.

Dabei gibt es im Moment widersprüchliche Signale in Bezug auf die Einordnung von rein digitalen Artefakten und digitalen Räumen. Vereinzelte Entscheidungen von Gerichten in Asien legen nahe, dass diese Räume als eine Art virtuelles Staatsgebiet zu sehen wären. Diese entschieden, dass virtuelle Ausrüstungsgegenstände in einem Computerspiel strafrechtlich relevant gestohlen werden konnten. ${ }^{6}$

Diese virtuellen Ausrüstungsgegenstände können in vollem Umfang ausschließlich in einem virtuellen Raum genützt werden. Auch mit Brückentechnologien wie dem 3D-Druck ist es nicht möglich, diese adäquat als physische Artefakte zu reproduzieren. Dies legt nahe, dass rein digitale Artefakte optimaler Weise in einer digitalen Umgebung analysiert und erhalten werden sollten. Digitale Artefakte sind als Primärquellen aufgrund ihrer Eigenschaften anspruchsvoll, denn sie sind fast untrennbar mit den Räumen verbunden, in denen sie existieren. Darüber hinaus sind sie relativ volatil, da sich die Räume und die Artefakte rasch ändern und das Artefakt leicht verloren gehen kann. Dies haben beispielsweise die Datenfehler bei MySpace im Jahr 2019 auf schmerzhafte Weise demonstriert: Durch den Verlust eines Backups ${ }^{7}$ gingen fast die gesamten dort gespeicherten Musikdateien und Inhalte der Seite unwiederbringlich verloren, denn auch das Internet Archive konnte nur 500.000 der über 50.000.000 Musikstücke wiederherstellen. ${ }^{8}$

3 Jason HEPPLER, What Is Digital Humanities?, online unter: https://whatisdigitalhumanities.com/ (09.04.2019).

4 LiNHD, Day of DH, online unter: https://web.archive.org/web/20180828230309/http://dayofdh2016.linhd.es/ (09.04.2019).

5 Siehe dazu beispielsweise: Henri Lefebvre, Die Produktion des Raums. The Production of Space, Leipzig 2018.

6 Eric Jou, Two Gamers Are Going To Jail For Stealing \$6,405 In Virtual Items, online unter: https://web.archive. org/web/20190409160834/https://kotaku.com/two-gamers-are-going-to-jail-for-stealing-6-405-in-vir-1571780985 (09.04.2019).

7 Siehe dazu beispielsweise: Cory Doctorow, Myspace lost all the music its users uploaded between 2003 and 2015 , online unter: https://web.archive.org/web/20190409162907/https://boingboing.net/2019/03/17/facebook-is-next.html (09.04.2019).

8 Internet Archive, The Myspace Dragon Hoard (2008-2010), online unter: https://archive.org/details/myspace_dragon_ hoard_2010 (09.04.2019). 


\section{Die unendliche Vielfalt der digitalen Quellen}

Der oben beschriebene Verlust digitaler Artefakte bei MySpace ist nur ein Beispiel für die Herausforderungen bei der Arbeit mit digitalen Quellen und deren Erhaltung, denn derer gibt es viele:

Der schiere Umfang der neu entstehenden Quellen ist kaum vorstellbar. Mit Oktober 2018 sind etwa 4,5 Milliarden Menschen im Internet aktiv - etwa 3,4 Milliarden davon sind Nutzer*innen sozialer Medien. So wurden laut einer Zusammenstellung von Domo ${ }^{9} \mathrm{im}$ Jahr 2018 in jeder einzelnen Minute 3,8 Millionen Suchanfragen von Google abgewickelt und die User senden 15 Millionen SMS, 46.000 Bilder via Instagram und 159 Millionen Emails (wobei etwa die Hälfte davon Spam ist). In jeder Minute fließen 3 Millionen GB an Daten über das Netz, 63 Websites werden gehackt und 2.500 MW werden zur Aufrechterhaltung der Infrastruktur verbraucht.

Neben dem reinen Umfang steigt auch die Komplexität der Erhaltung. Denn zur Erhaltung eines Artefaktes muss oft auch der digitale Raum miterhalten werden. Dies beinhaltet die Erhaltung und Reproduktion der zugrundeliegenden Soft- und Hardware. Darüber hinaus gibt es oft physische Beigaben (z.B. Handbücher) und verwandte digitale Quellen, die Metainformationen über das eigentliche Artefakt enthalten (z.B. Diskussionsforeneinträge, Podcasts, Videos) und mit inm erhalten und untersucht werden sollten (siehe Abb. 1).

\section{Elemente digitaler Artefakte}

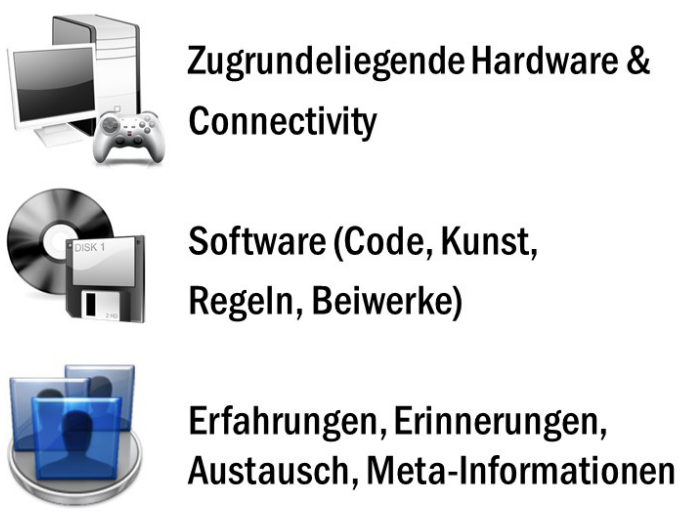

Abb. 1: Elemente digitaler Artefakte. Hausar 2019.

\section{Digital Humanities to the rescue?}

Die Digital Humanities bewegen sich in diesem Meer aus Code und Daten im Rahmen des digitalen Ökosystems. Sie leisten eine doppelte Arbeit indem sie den Brückenschlag zwischen den seit Jahrhunderten analog aufbewahrten Quellen und den originär digitalen Quellen versuchen, um eine Analyse primär analoger und digitaler Quellen in einheitlicher, digitaler Form zu gewährleisten. Dabei ist dieser Schritt auch eine Flucht nach vorne, da eine Analyse und Aufarbeitung ganz ohne digitale Werkzeuge fast nicht mehr möglich erscheint.

9 Domo, Data Never Sleeps 6.0, online unter: https://web.archive.org/web/20190409161923/ bzw. https://web-assets. domo.com/blog/wp-content/uploads/2018/06/18-domo-data-never-sleeps-6.png (09.04.2019). 
Diese Werkzeuge sind auch bei der ,Übersetzung und weitergehenden Erschließung von (bereits aufgearbeiteten) analogen Quellen in digitale im Einsatz, die im Moment stark im Fokus der Forschung ist. Es ist daher auch nicht verwunderlich, dass digitale Elemente sich hauptsächlich über den Einsatz digitaler Werkzeuge in die Arbeit digitaler Humanisten ,einschreiben' und gerade in den Anfängen weniger Aufmerksamkeit auf den primär digitalen Quellen ruht.

Dies lässt sich auch gut über Begriffsvergleiche in Google Books ngram-viewer visualisieren. Während der Begriff „Humanities Computing“, der seit den frühen Siebzigerjahren in Gebrauch ist, stärker den Einsatz digitaler Rechenmaschinen referenziert, kommen in englischsprachigen Büchern seit den Neunzigerjahren vermehrt Begriffe auf, die weniger auf die Werkzeuge und mehr auf Eigenschaften bzw. Räume hinweisen. Der Gebrauch der Begriffe „Digital Archaeology“, „Digital History“ und seit dem Jahr 2000 auch vermehrt „Digital Humanities“ nimmt zu, während „Humanities Computing“ als Begriff weniger oft genutzt wird (siehe Abb. 2).

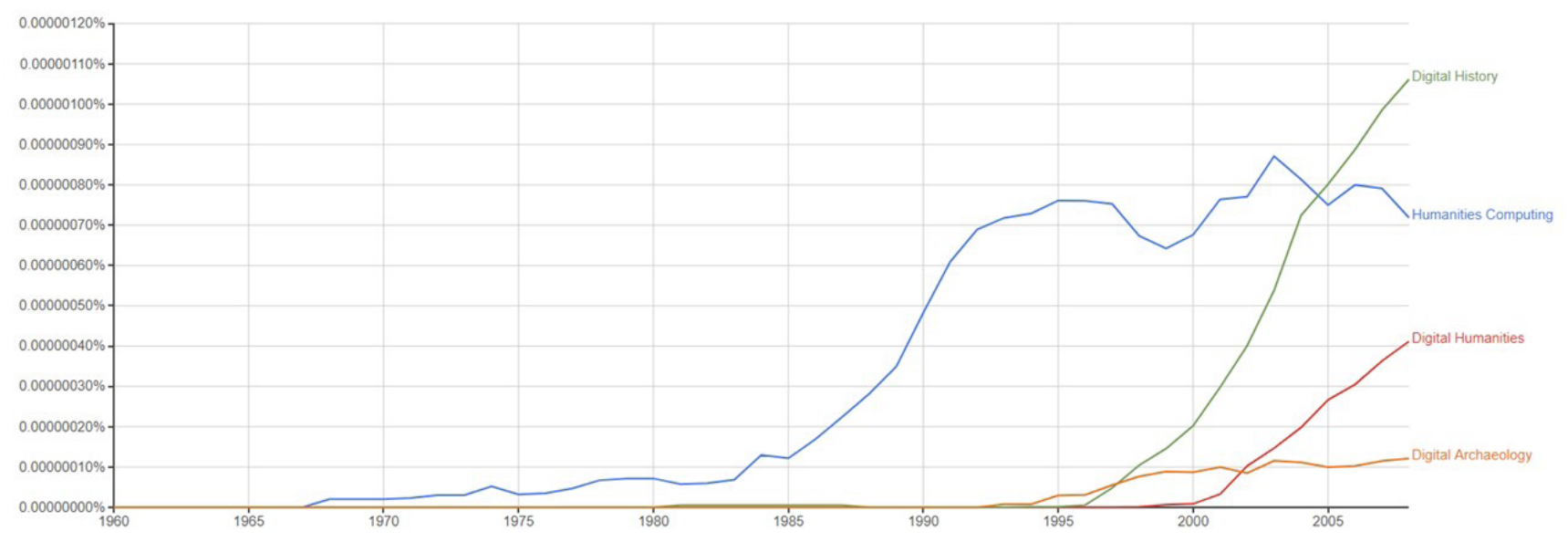

Abb. 2: Häufigkeit der Digital Humanities Begriffe im Vergleich. Google Books ngram Viewer (English Corpus, Smoothing 3). Hausar 2019.

\section{Die Kurzlebigkeit digitaler Quellen}

Der Erhalt primär digitaler Quellen ist manchmal sehr anspruchsvoll und gleicht dem Versuch, in warmem Klima langfristig Schneebälle in Kühlschränken aufzubewahren. Daher ist es auch durchaus verständlich, dass es kaum dezentrale und noch weniger zentrale umfassende Ansätze zur Archivierung der digitalen Primärquellen gibt, sodass wir von der Quellenlage her de facto seit den 1980er Jahren ein schwarzes Loch haben, welches durch "Citizen Scientists and Archivars“ (archive.org, Computermuseen, Hackerkollektive, Bootleg Archive etc.) sowie institutionelle oder regionale Initiativen (z.B. Phaedra an der Universität Wien bzw. ARCHE am Austrian Center für Digital Humanities der ÖAW) in geringem Umfang abgefedert wird.

Die Bemühungen von Citizen Scientists umfassen meist nur relativ statische Räume (z.B. Newsgroups, eMails, statische Websites, Single Player Games, Videos etc.), massive virtuelle Onlineumgebungen wie beispielsweise VR-Chatwelten (z.B. das immer noch existierende Second Life), Soziale Plattformen (Facebook, YouTube, Snapchat) oder Computerspiele wie MMRPGs (EVE Online, World of Warcraft etc.) sind hier noch gar nicht inkludiert. 
Schwierigkeiten bei der Sichtung bereiten dabei die sich ständig wandelnden Formen des digitalen Status quo bei dynamischen digitalen Quellen, die eine permanente Versionierung aller Artefakte erfordern würde und außerdem auch noch von der jeweiligen Hardware abhängt. Erst dann könnte man beispielsweise eine Begegnung mehrerer Avatare auf einer sozialen Plattform oder eine Partie Tetris mit mehreren Spieler*innen, die gegeneinander angetreten sind und sich dabei über Chatsysteme auch unterhalten haben, archivieren und über das Archiv auch langfristig ,erleben' und erforschen. Dieses Problem kann hier nicht weiter erläutert werden, allerdings ist es der wichtigste ungeklärte Punkt für langfristige Erforschung und Erhaltung dynamischer digitaler Artefakte, für die bis jetzt noch keine funktionierenden methodischen oder praktischen Konzepte formuliert - und noch viel weniger umgesetzt - werden konnten.

Hier wären die Nationalbibliotheken als jene Institution, die einen nationalen, gesetzlichen Archivierungsauftrag haben, gefordert, die ähnlich wie das privat ins Leben gerufene und von staatlichen Stellen in den USA unterstützte Archive.org diese Aufgabe mit neuer Finanzierung über digitale Medienarchive erfüllen müssten.

Besondere Herausforderungen für sowohl staatliche als auch private digitalen Archivare ergeben sich in den nachfolgenden vier großen Bereichen:

- Legal: Rechtslage teilweise ungeklärt und widersprüchlich, Lizenzen falsch gewählt

- Software: DRM \& Kopierschutz, Abandonware, Betriebssysteme, proprietäre Formate

- Hardware: Peripheriegeräte, Laufwerke, VR-Brillen, diverse Endgeräte, Lagerraum

- Kosten: Initialkosten sowie Kosten zur langfristigen Erhaltung und der Aufrechterhaltung in betriebsfähigen Zustand, Lagerräume

Insbesondere im Rechtsbereich besteht Handlungsbedarf, da dieser den Rahmen für alle Archivierungsbemühungen vorgibt. Denn das klassische Verständnis des Urheberrechts als Ausnahme des Rechts der Allgemeinheit auf Information, Kultur, Bildung und Forschung sowie eines gemeinsamen Bewahrens von Gemeingütern wird zugunsten von Individualrechten und einem absoluten Urheberrechtsansatz zurückgedrängt. Dies bringt Forscher*innen in unhaltbare Rollen.

Diese heute mehr und mehr vorherrschende Sicht gibt Verwerter*innen (Autor*innen bzw. jenen, denen sie die Rechte übertragen haben) eine fast absolute Kontrolle über Inhalte. Dieser Logik folgend hätten wohl Kafkas Werke seinem Wunsch nach verbrannt und nie verlegt werden dürfen - und das ist nur ein Beispiel für den Schaden, den diese von wirtschaftlichen Überlegungen dominierte Herangehensweise ohne ausgewogene Regulierung anrichten kann.

Für die Erforschung ist es unbedingt notwendig, aktiv und zeitnah gerade digitale Artefakte zu archivieren, damit sie überhaupt erforscht werden können. Dies wird durch rechtliche Konstrukte allerdings unmöglich gemacht. Dies wird beispielsweise auch dann deutlich, wenn der deutsche Gesetzgeber explizit ein Recht auf Knacken des Kopierschutzes für die Nationalbibliothek aussprechen muss, um zumindest einige wenige Artefakte rechtmäßig erhalten zu können. Aber selbst dieses Recht umfasst nur die Information, nicht auch die nötige Hardware, um die Information abrufen zu können. 


\section{Retten, was zu retten ist}

Es gäbe in Bezug auf die Erforschung und die damit unmittelbar verbundene Archivierung primär digitaler Quellen noch viel zu sagen, insbesondere auch zum Selbstverständnis und der Praxis von „digital natives“, dem Graubereich der Abandonware, illegalen Archiven, privaten Sammler*innen und der unfreiwilligen Archivfunktion die sogar digitalen Produktpirat*innen zukommen könnte. Dies würde allerdings den Rahmen sprengen und soll deshalb nur als Anregung für weitere Forschungen kurz erwähnt werden.

Trotzdem gibt es abschließend drei einfache Handlungsanweisungen bei digitalen Forschungsprojekten, die Analyse und Archivierung wesentlich erleichtern, wenn sie mitbedacht werden:

- Daten auch in privaten Archiven ablegen: Jedenfalls auch eine private Archivierung bei Projekten einplanen (e.g. archive.org, archiveteam.org)

- Koordination der Archivierung aktiv in Angriff nehmen: Informationen mit Archiven teilen und Archivierung koordinieren, Archivierung mitdenken

- Teilen von Informationen als zusätzliche Kulturtechnik der Langzeiterhaltung: Informationen öffentlich und an mehreren Stellen unter Open Access-Lizenzen online stellen (inkl. Anleitungen, Zusatzdaten etc.)

In diesem Sinne - und auch unter Berücksichtigung der Masse zu archivierender Daten - ist die Lage hoffnungslos, aber nicht ernst.

\section{Literaturverzeichnis}

Cory Doctorow, Myspace lost all the music its users uploaded between 2003 and 2015, online unter: https://web. archive.org/web/20190409162907/https://boingboing.net/2019/03/17/facebook-is-next.html (09.04.2019).

Domo, Data Never Sleeps 6.0, online unter: https://web.archive.org/web/20190409161923/https://web-assets. domo.com/blog/wp-content/uploads/2018/ (09.04.2019).

Gernot HAUSAR, Weitere Überlegungen zu digitalen Geschichts- und Geisteswissenschaften, in: Julia Menzel, Hg., Digitalität - Theorien und Praktiken des Digitalen in den Geisteswissenschaften 2016, online unter: https://web.archive.org/web/20190409173203/https://digigeist.hypotheses.org/308 (09.04.2019).

Jason HePpler, What Is Digital Humanities?, online unter: https://whatisdigitalhumanities.com/ (09.04.2019).

Internet Archive, The Myspace Dragon Hoard (2008-2010), online unter: https://archive.org/details/myspace dragon_hoard_2010 (09.04.2019).

Eric Jou, Two Gamers Are Going To Jail For Stealing \$6,405 In Virtual Items, online unter: https://web.archive. org/web/20190409160834/ bzw. https://kotaku.com/two-gamers-are-going-to-jail-for-stealing-6-405-invir-1571780985 (09.04.2019).

Henri Lefebvre, Die Produktion des Raums. The Production of Space, Leipzig 2018.

LiNHD, Day of DH, online unter: https://web.archive.org/web/20180828230309/http://dayofdh2016.linhd.es/ (09.04.2019).

Wolfgang ScHmaLe, Digitale Geschichtswissenschaft, Köln / Wien 2010. 